\title{
Erratum to: Query-Based Extractive Text Summarization for Sanskrit
}

\author{
Siddhi Barve, Shaba Desai and Razia Sardinha
}

Erratum to: S. Das et al. (eds.), Proceedings

of the 4th International Conference on Frontiers

in Intelligent Computing: Theory and Applications

(FICTA) 2015, Advances in Intelligent Systems

and Computing 404, DOI 10.1007/978-81-322-2695-6_47

Erratum DOI: 10.1007/978-81-322-2695-6_62

In original version of this chapter Tables 1-4 consist of scrambled texts that have been corrected now.

2. The preprocessing steps are performed on the selected document. The steps include sentence length feature, tokenization, stop word removal, morphological analysis, and sandhi and compound resolution.

Below is an illustration of morphological analysis and sandhi/compound resolution for the document "कदली."

The online version of the original chapter can be found under 10.1007/978-81-322-2695-6_47

\footnotetext{
S. Barve $(\bowtie) \cdot$ S. Desai · R. Sardinha

Department of Information Technology, Padre Conceicao College of Engineering,

Goa University, Goa, India

e-mail: barvesiddhi@gmail.com

S. Desai

e-mail: shaba.desai@gmail.com

R. Sardinha

e-mail: razia.sardinha@gmail.com 
- Morphological Analysis

Analysis for the word "आम्र" follows:

आम्न = आम्न \{पुं $\}\{2$;एक $\}$

आम्मं $\{$ नपुं $\}\{1$ एक $\}$

आमं \{नपुं $\{2$;एक $\}$

- Sandhi/Compound Resolution

Suppose if the encountered word, "रक्तवर्णीयम्" is compound, then the split can be obtained as रक्तवर्णीयम्= रक्त-वर्णि-इयम् .

The word obtained could also be a Sandhi; if it is Sandhi, then the splits are obtained as चेदपि = चेत्+अपि .

After preprocessing is carried out, the user is prompted to enter the query corresponding to the document. The query is "वृक्षाः कदली शलाटुः फलं."

Table 1 Raw Sanskrit document

आम्रं पनसं, कदलीफलं च प्रमुखफलत्रयत्वेन परिगण्यन्ते । एवम् एव कदली-आम्र-उदुम्बर तिन्त्रिणीवृक्षाः प्रमुखाः वृक्षाः इति उच्यन्ते ।एतान् अधिकृत्य काचित विचित्रा कथा केषुचित प्रदेशेषुश् श्रूयते ।एते पर्च वृक्षाः अपि कस्मिंश्चित् काले मनुष्यरुपेण सहोदर्यः आसन् ।बहुकालं यावत् तासां विवाहः न जातः |कदाचित् देवः प्रत्यक्षीभूय 'विवाहम् इच्छन्ति वा ?' इति ताः पॄष्टवान् ।तदा चतस्रः सहोदर्यः विवाहम् अङ्घीकृतवत्यः । किन्तु अन्तिमा सहोदरी न अङ्गीकृतवती । सा सन्तानमात्रं प्रार्थितवती । अनन्तरं देवः ताः सर्वाः वृक्षरुपेण परिवर्त्य - "यः एतान् वृक्षान् आरोहति सः एव पतिः ' इति उक्तवान् । अतः एव् विवाहम् अङ्गीकृतवतः वृक्षान सर्वे आरोढुं शक्नुअन्ति चेदपि कदलीवृक्षं कोऽपि आरोढ़ं न शक्नोति । कदली इति संस्कृतभाषया, हिन्दिभाषया केला इति, आंगलभाषया बनाना प्लाण्टन, आडम्स् आपिल इत्यपि उच्यते। काभिश्चित् भारतीयभाषाभिः कदलीइत्येव उच्यते ।तमिळु मलयाळभाषया च वाळ्:ऐ इति, तेलुगुभाषया अरटि इति च उच्यते । बौद्धाः कदलीवृक्षं पवित्रं मन्यन्ते । बौद्धसाहित्ये उक्तं मोचापानं कदलीफलेन एव सज्जीक्रियते । अस्माकं पुराणे श्रूयते यत् -हनुमान् हिमालयप्रान्ते कदलीवने आसीत् इति । कदलीवृक्षस्य पुष्पं, शलाटु:, फलं च आहररुपेण उपयुज्यते । कदलीवृक्षस्य शलाटुः हरितवर्णीयः भवति। तस्य फलं हरितं ,पीतं, पीतहरितमिश्रितं, रक्तवर्णीयं वा भाति । तदा आकारेण लघु बृहत् चापि भवति । कदल्यां ५,००० प्रभेदाः सन्ति इति श्रूयते । पच्चेकदली, बूदिकदली, वृक्षकदली, रसकदली, एलाकदली, अरण्यकदली इति अष्ट, दश वा विधाः प्रसिद्धाः सन्ति । कदलीपर्ण बृहदाकारकं पञ्चषपादमितदीर्धम्, अधिकविशालं च भवति । दक्षिणाभारते केषचित स्थलेषु भोजनार्यम् एतस्य उपयोगः क्रियते। जनाः विवाहादिशुभकार्येषु वितानं मण्डपं द्वारं च पुष्पगुच्छसहितेन कदलीवृक्षेण अलङ्कुर्वन्ति । एतं शुभस्केतं मन्येन्ते जनाः । पक्वानि कदलीफलानि कदलीपुष्पम् यदा शलाटुः पक्वं भवति तदा वृक्षं कर्तयन्ति । कर्तितवृक्षस्य प्रकाण्डं परितः स्थितेभ्यः कन्देभ्यः नूतनसस्यानि उत्पद्यन्ते । एवं किदलीसन्तानः वर्धते । 
Table 2 Summary generated by average tf.isf approach

\begin{tabular}{l} 
आम्मं पनसं, कदलीफलं च प्रमुख फल त्रयत्वेन परिगण्यन्ते । एवम् एव कदली-आम्र-उदुम्बर \\
तिन्त्रिणीवृक्षाः प्रमुखाः वृक्षाः इति उच्यन्ते । अतः एव् विवाहम् अड्ग्वीकृतवतः वृक्षान सर्वे आरोढुं श \\
क्नुअन्ति चेत् अपि कदलीवृक्षं कः अपि आरोढुं न शक्नोति । कदली इति संस्कृतभाषया, \\
हिन्दिभाषया केला इति, आंगलभाषया बनाना प्लाण्टन्, आडम्स् आपिल इति अपि उच्यते । अस्माकं \\
पुराणे श्रूयते यत् हनुमान् हिमालयप्रान्ते कदलीवने आसीत् इति । प-च्चेकदली, बूदिकदली, वृक्ष- \\
कदली, रस-कदली, एला-कदली, अरण्य-कदली इति अष्ट, दश वा विधाः प्रसिद्धाः सन्ति । \\
\hline \hline
\end{tabular}

Table 3 Summary generated by graph-based approach

कदलीवृक्षस्य शलाटुः हरितवर्णीयः भवति | कदलीपर्णं बृहदाकारकं पঙ्चषप अदमितदीर्धम्, अधिक
विशालम् च भवति| बौद्धाः कदली-वृक्षं पवित्रं मन्यन्ते । कदलीवृक्षस्य पुष्पं, शलाटुः, फलं च
आहररुपेण उपयुज्यते । काभिः चित् भारतीयभाषाभिः कदली इति एव उच्यते | पक्वानि
कदलीफलानि कदली-पुष्पम् यदा शलाटुः पक्वं भवति तदा वृक्षं कर्तयन्ति।

Table 4 Summary generated by VSM approach

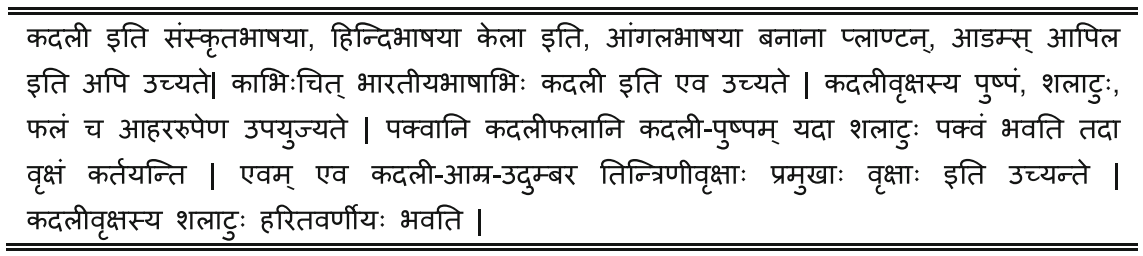

